Pacific Journal of Mathematics

CENTRALIZERS OF IRREGULAR ELEMENTS IN REDUCTIVE 


\title{
CENTRALIZERS OF IRREGULAR ELEMENTS IN REDUCTIVE ALGEBRAIC GROUPS
}

\author{
JOHN F. KURTZKE, JR.
}

Let $G$ be a reductive linear algebraic group defined over an algebraically closed field $K$. An element $x \in G$ is called regular if $\operatorname{dim} Z_{G}(x)$ is the rank of $G$ - which is the smallest possible dimension for a centralizer - otherwise $x$ is called irregular. T. A. Springer has shown that if $x \in G$ is regular, then $Z_{G}(x)^{0}$ is abelian. In this paper, we show that when char $K$ is good for $G$, the converse is also true - if $x \in G$ is irregular, then $Z_{G}(x)^{0}$ is nonabelian. In the course of the proof of this, we show that if $G$ is a classical group or $G_{2}$ (char $K \operatorname{good}$ ), then $\operatorname{dim} Z\left(Z_{G}(x)^{0}\right)$ is at most rank $G$. Further, if $G=S p_{2 n}(K)(\operatorname{char} K \neq$ 2), then $Z\left(Z_{G}(x)^{0}\right)$ consists of polynomials in $x$.

Introduction and statement of results. In this paper, all groups will be linear algebraic groups defined over an algebraically closed field $K$. If $G$ is a group, then $G^{0}$ denotes its identity component and $\mathfrak{g}$ or $\mathscr{L}(G)$ denotes the Lie algebra of $G$. If $G$ is reductive (we require that reductive groups be connected), then by "the simple components of $G$ ", we mean the simple components of the semisimple group $G / \operatorname{Rad} G$ where $\operatorname{Rad} G$ is the radical of $G$.

Let $G$ be a reductive group. An element $x \in G$ is called regular if $\operatorname{dim} Z_{G}(x)$ is minimal in $\left\{\operatorname{dim} Z_{G}(y) \mid y \in G\right\}$; otherwise, $x$ is called irregular. The regular elements are dense in $G$; the identity element is always irregular. In $G L_{n}(K)$, a diagonalizable element is regular if and only if all its eigenvalues are distinct. It is well known that if $x \in G$ is regular, then

$$
\operatorname{dim} Z_{G}(x)=\operatorname{rank} G
$$

and if $y \in G$ is irregular, then

$$
\operatorname{dim} Z_{G}(y) \geq 2+\operatorname{rank} G
$$

(cf. [17]). If

$$
\operatorname{dim} Z_{G}(y)=2+\operatorname{rank} G,
$$

then $y$ is called subregular.

T. A. Springer has shown that if $x \in G$ is regular, then $Z_{G}(x)^{0}$ is abelian. [13] Under some very mild restrictions on char $K$ (char $K=0$ or char $K \geq 7)$, we shall prove the converse; namely, that if $Z_{G}(x)^{0}$ is 
abelian, then $x$ is regular - or, equivalently, if $x \in G$ is irregular, then $Z_{G}(x)^{0}$ is nonabelian. This last statement is the one that we will actually prove.

Our proof uses the classification of nilpotent conjugacy classes in a simple Lie algebra, and since this classification is only valid in good characteristics, we must require that char $K$ be good for $G$. The usual way of defining good characteristic for a reductive group $G$ (or Lie algebra $g$ ) is to define a bad prime as one that divides a coefficient in the highest root. Then all other primes and zero are called good. If $G$ is of type $A_{n}$, then all characteristics are good. So char $K$ is good for $G$

(a) if $G$ has a component of type $B_{n}, C_{n}$, or $D_{n}$, then char $K \neq 2$

(b) if $G$ has a component of type $G_{2}, F_{4}, E_{6}$, or $E_{7}$, then char $K \neq 2$ or 3

(c) if $G$ has a component of type $E_{8}$, then char $K \neq 2,3$, or 5 . Hence, if char $K=0$ or char $K \geq 7$, then char $K$ is always good.

THeOREM A. Let $G$ be a reductive group with char $K$ good for $G$. Then $x \in G$ is regular if and only if $Z_{G}(x)^{0}$ is abelian.

Proving Theorem A is the main object of this paper. We will first make some reductions (§2), then handle the classical groups (§3) and finally the exceptional groups ( $\$ \$ 4$ and 5$)$. In the course of this, we will derive some stronger results for the classical groups. We say that a reductive group $G$ is of classical type if none of its components is an exceptional group.

THEOREM B. 1. Let $G$ be a group of classical type with char $K \neq 2$. Let $x \in G$. Then

$$
\operatorname{dim} Z\left(Z_{G}(x)^{0}\right) \leq \operatorname{rank} G .
$$

Equality holds if and only if $x$ is regular.

2. Let $G=G_{2}$ with char $K \neq 2$ or 3 and let $x \in G$. Then

$$
\operatorname{dim} Z\left(Z_{G}(x)^{0}\right) \leq \operatorname{rank} G .
$$

Equality holds only in two cases.

(i) $x$ is regular, or

(ii) $x$ is subregular, but not semisimple.

The first part is proved in $\S 3$; the second part in $\S 5$. Note that $\operatorname{dim} Z\left(Z_{G}(x)^{0}\right)$ can equal rank $G$ even if $x$ is irregular (we suspect that $G_{2}$ 
is the only simple group in which this can happen). For the symplectic groups, we can improve Theorem B:

TheOREM C. Let $G=S p_{2 n}(K)$ with char $K \neq 2$ and let $x \in G$. Then $Z\left(Z_{G}(x)^{0}\right)$ contains only polynomials in $x$.

This is proved in $\S 3$. If $G=P G L_{2}(\mathrm{C})$ and

$$
x=\left[\begin{array}{rr}
1 & 0 \\
0 & -1
\end{array}\right]
$$

we see that $x$ is regular, but $Z_{G}(x)$ is nonabelian - of course $Z_{G}(x)^{0}$ is abelian. This happens because $Z_{G}(x)$ is not connected, and that happens because $G$ is not simply connected (as $x$ is semisimple). Now if $G$ is semisimple and simply connected, and if $x \in G$ is regular semisimple, then $Z_{G}(x)$ is connected and thus abelian. In her dissertation [9], B. Lou proved that if $u$ is a regular unipotent element in a semisimple group $G$, then $Z_{G}(u)$ is abelian (char $K$ arbitrary). Therefore, if $G$ is a semisimple, simply connected group, and if $x \in G$ is regular, then $Z_{G}(x)$ is abelian. So as a corollary to Theorem $\mathrm{A}$ we have:

THEOREM D. Let $G$ be a semisimple, simply connected group with char $K$ good for $G$. Then $x \in G$ is regular if and only if $Z_{G}(x)$ is abelian.

As general references to algebraic groups and Lie algebras, we cite Humphreys' books [7, 7a] which we follow for basic definitions and conventions. For conjugacy classes and centralizers, the reader is invited to consult the Springer-Steinberg article [16] or Steinberg's Tata notes [18]. The classification of unipotent and nilpotent conjugacy classes is described in papers of Dynkin [4], Elkington [6], and Bala-Carter [1, 2]. The extension of the Dynkin classification to all good characteristics is described by Springer-Steinberg [16] for the classical groups, by Jeurissen and Stuhler [19] for $G_{2}$, and by Pommerening [10, 11] for the other exceptional groups. Dynkin, Elkington, and Bala-Carter also have information about the centralizers of nilpotent elements, which can also be found in an article of Elasvili [5].

2. Reductions. In this section, we show that in order to prove Theorem A, it suffices to prove:

THEOREM $\mathfrak{a}$. Let $\mathrm{g}$ be a simple Lie algebra with char $K$ good for $\mathrm{g}$. If $X \in \mathfrak{g}$ is nilpotent and irregular, then $\mathrm{z}_{\mathrm{g}}(X)$ is nonabelian. 
The rest of this paper will then be devoted to proving Theorem $a$. The first thing we notice is that if $G$ is reductive and $t \in G$ is semisimple, then $Z_{G}(t)^{0}$ is well in hand:

Proposition 2.1. Let $G$ be a reductive group and let $t \in G$ be semisimple. Let $T$ be a maximal torus for $G$ containing $t$ and let $\Psi$ be the resulting set of roots. Then

1. $Z_{G}(t)^{0}$ is generated by $T$ and the one parameter unipotent subgroups $U_{\alpha}$ for those $\alpha \in \Psi$ for which $\alpha(t)=1$. Moreover, $Z_{G}(t)^{0}$ is reductive and has the same rank as $G$.

2. $Z_{G}(t)$ is generated by $Z_{G}(t)^{0}$ and the $n \in N_{G}(T) / T$ such that $n t n^{-1}=t$.

3. $t$ is regular if and only if $\alpha(t) \neq 1$ for all $\alpha \in \Psi$

4. $\operatorname{dim} Z\left(Z_{G}(t)^{0}\right) \leq \operatorname{rank} G$

Equality occurs if and only if $t$ is regular.

This is rather well known and the proof can be found in [18] and [3]. If we were dealing with real compact semisimple Lie groups, then we would be finished here. It is the unipotent elements of $G$ that are the obstacle.

If $G$ is reductive, and $x \in G$ is arbitrary, then we can write $x=s u$ with $s$ and $u$ commuting semisimple and unipotent elements of $G$ respectively (Jordan decomposition). If we let $H=Z_{G}(s)^{0}$, we see that $u \in H$ and $Z_{G}(x)^{0}=Z_{H}(u)^{0}$. Because the ranks of $G$ and $H$ are the same, we see that $x$ is regular in $G$ if and only if $u$ is regular in $H$. So we have:

Remark 2.2. To prove Theorem A, it suffices to assume that the element $x$ is unipotent.

The next step is to reduce the problem to the case where the group $G$ is simple. Further we will only have to look at isogeny classes of simple groups instead of isomorphism classes.

Proposition 2.3. Let $G$ be a reductive group, $R$ a closed normal subgroup contained in the center, and $x \in G$. Let $\pi: G \rightarrow G / R=H$ be the canonical quotient map with $\pi(x)=\bar{x}$. Then

1. $R \cap(G, G)$ is finite and $R^{0}$ is a torus.

2. $\operatorname{dim} Z_{G}(x)=\operatorname{dim} R+\operatorname{dim} Z_{H}(\bar{x})$.

Thus $x$ is regular in $G$ if and only if $\bar{x}$ is regular in $H$.

3. $\operatorname{dim} Z\left(Z_{G}(x)^{0}\right)=\operatorname{dim} R+\operatorname{dim} Z\left(Z_{H}(\bar{x})^{0}\right)$.

Thus the conclusions of Theorems $\mathrm{A}$ and $\mathrm{B}$ hold for $G$ if and only if they hold for $H$. 
Proof. 1. This is standard; see [7a], for example.

2. Let $\bar{C}=Z_{H}(\bar{x})$ and $C=\pi^{-1}(\bar{C})$. We have to show that $\operatorname{dim} C=$ $\operatorname{dim} Z_{G}(x)$. Now $y \in C$ if and only if $x y x^{-1} y^{-1} \in R$. As $R \cap(G, G)$ is finite, so is $R \cap\left\{x y x^{-1} y^{-1} \mid y \in G\right\}$. Let $\left\{r_{1}, \ldots, r_{p}\right\}$ be that set and choose $y_{l} \in G$ so that $x y_{i} x^{-1} y_{i}^{-1}=r_{i}$. Now $x y x^{-1} y^{-1}=x z x^{-1} z^{-1}$ precisely when $z^{-1} y \in Z_{G}(x)$, so the $y_{i}$ are determined $\bmod Z_{G}(x)$. Therefore, $C=$ $\cup_{i=1}^{p} y_{i} Z_{G}(x)$ and $C^{0}=Z_{G}(x)^{0}$ as desired.

Let $\bar{Z}_{1}=Z\left(Z_{H}(\bar{x})^{0}\right)$ and $Z_{1}=\pi^{-1}\left(\bar{Z}_{1}\right)^{0}$. Clearly

$$
Z\left(Z_{G}(x)^{0}\right)^{0} \subseteq Z_{1} \subseteq Z_{G}(x)^{0} .
$$

We want to show that the first inclusion is an equality. Choose $y \in Z_{1}$. Now $\left(y, Z_{G}(x)^{0}\right)$ is a finite set, contains 1 (as $y$ commutes with $x$ ) and is connected. Hence $y \in Z\left(Z_{G}(x)^{0}\right)$. As $Z_{1}$ is connected, we have $y \in$ $Z\left(Z_{G}(x)^{0}\right)^{0}$ as desired.

ReMARK 2.4. 1. Let $G$ be reductive. Then the conclusions of Theorems A and B hold for $G$ if and only if they hold for $G / \operatorname{Rad} G$. So we can assume in Theorems A and B that $G$ is semisimple.

2. Let $G$ and $H$ be isogenous semisimple groups. Then the conclusions of Theorems A and B hold for $G$ if and only if they hold for $H$.

3. To prove Theorems $\mathrm{A}$ and B, we may assume that $G$ is simple and $x$ is unipotent. Further, it is enough to prove these theorems for one group in each simple isogeny class.

Proof. 1. Let $R=\operatorname{Rad} G$ and apply Proposition 2.3.

2. Let $\pi_{1}: \tilde{G} \rightarrow G$ and $\pi_{2}: \tilde{G} \rightarrow H$ be the universal covers of $G$ and $H$. Now apply Proposition 2.3.

3. We can assume that $G$ is adjoint and semisimple. Then $G=\prod_{i=1}^{r} G_{i}$ is a direct product of simple groups. If $x \in G$, then $x=x_{1} \cdots x_{r}$ with $x_{\imath} \in G_{i}$. So $Z_{G}(x)^{0}=\prod_{i=1}^{r} Z_{G_{i}}\left(x_{i}\right)^{0}$.

The next task is to transfer all this to the Lie algebra. If $x \in G$ and $X \in \mathfrak{g}$, we want to use results about $z_{\mathrm{g}}(X)$ to understand $Z_{G}(x)^{0}$. If char $K=0$, then $\mathcal{L}\left(Z_{G}(x)^{0}\right)={ }_{z_{g}}(x)$ and $\mathcal{L}\left(Z_{G}(X)^{0}\right)=z_{\mathfrak{g}}(X)-$ if $x$ and $X$ are semisimple, then these hold no matter what char $K$ is. When $K=\mathbf{C}$, then there are maps ( $\log$ and exp) from $G$ to $g$ and vice versa which are power series and local isomorphisms of manifolds. So when $K=\mathbf{C}$ and $\log x=X$, then we have $\mathcal{L}\left(Z_{G}(x)^{0}\right)=z_{\mathrm{g}}(x)=z_{\mathrm{g}}(X)=\mathcal{L}\left(Z_{G}(X)^{0}\right)$ which is as nice a relation among centralizers as one could want. We would like this to happen in our case $-G$ reductive and $x$ unipotent. Because we have room in the isogeny classes to skip around and because char $K$ is good (by assumption), we can actually do this. 
Proposition 2.5 (Richardson). Let $G$ be a reductive group with char $K$ good for $G$. Let $x \in G$ and $X \in \mathfrak{g}=\mathcal{L}(G)$. Then

$$
\mathcal{L}\left(Z_{G}(x)^{0}\right)=z_{\mathfrak{g}}(x)
$$

and

$$
\mathcal{L}\left(Z_{G}(X)^{0}\right)=z_{g}(X) \text {. }
$$

The proof is in [12]. Next, we let $U=\{x \in G \mid x$ is unipotent $\}$, the unipotent variety, and $\mathfrak{n}=\{X \in \mathfrak{g} \mid X$ is nilpotent $\}$, the nilpotent variety.

Proposition 2.6. (Springer). Let $G$ be a semisimple, simply connected group with char $K$ good for $G$. Then there is a G-equivariant bijective morphism $f: V \rightarrow \mathfrak{n}$ which topologically is a homeomorphism.

The proof is in [15]. By $G$-equivariant, we mean that for $g \in G$ and $v \in V, \operatorname{Ad} g(f(v))=f\left(g v g^{-1}\right)$. Thus $f$ preserves centralizers and if $f(x)=$ $X$, then $z_{g}(x)=\mathcal{L}\left(Z_{G}(x)^{0}\right)=\mathcal{L}\left(Z_{G}(X)^{0}\right)=z_{g}(X)$. It now makes sense to define regular, subregular, and irregular elements in reductive Lie algebras. In good characteristics, the results are the same as in reductive groups.

The final thing we have to notice is that in going from a group to its Lie algebra, the center can only get larger. That is, $\mathcal{L}\left(Z(G)^{0}\right) \subseteq z(g)$. Thus, in our case, if $z_{g}(X)$ is nonabelian, then so is $Z_{G}(X)^{0}$ and $Z_{G}(x)^{0}$.

Remark 2.7. To prove Theorem A, it is sufficient to prove Theorem a.

Proposition 2.8. (Steinberg, Springer). 1. Let $G$ be a reductive group with char $K$ arbitrary. Let $x \in G$ be unipotent and let $\Psi$ be a set of roots for $G$. Choose a base for $\Psi$ so that $x$ is supported by positive roots, i.e. $x=\prod_{\alpha>0} \varepsilon_{\alpha}\left(c_{\alpha}\right)$ where $c_{\alpha} \in K$. Then $x$ is regular if and only if $c_{\alpha} \neq 0$ for $\alpha$ simple. Further, all regular unipotent elements are conjugate.

2. Let $\mathrm{g}$ be a reductive Lie algebra with char $K$ good for $\mathrm{g}$. Let $X \in \mathrm{g}$ be nilpotent and let $\Psi$ be a set of roots for $\mathrm{g}$. Choose a base for $\Psi$ so that $X$ is supported by positive roots, i.e. $X=\Sigma_{\alpha>0} b_{\alpha} e_{\alpha}$ with $b_{\alpha} \in K$. Then $X$ is regular if and only if $b_{\alpha} \neq 0$ for $\alpha$ simple. All regular nilpotent elements are conjugate.

Part 1 is proved in [17]; part 2 in [14]. In particular $\prod_{\alpha \in \Delta} \varepsilon_{\alpha}(1)$ and $\Sigma_{\alpha \in \Delta} \varepsilon_{\alpha}$ are regular $(\Delta$ is a base of $\Psi$ ). These are called the standard regular and nilpotent elements respectively. 
3. The classical groups. In this section, we will prove Theorems A, B, and $\mathrm{C}$ for the classical groups. The next result is well-known.

Proposition 3.1. Let $G=G L_{n}(K)$ or $S L_{n}(K)$ and let $x \in G$. Then, 1. $Z\left(Z_{G}(x)\right)=\{$ polynomials in $x\} \cap G$. Therefore,

$$
\operatorname{dim} Z\left(Z_{G}(x)\right) \leq \operatorname{rank} G
$$

and equality holds if and only if $x$ is regular.

2. $Z\left(Z_{G}(x)^{0}\right)$ consists of polynomials in $x$. Therefore,

$$
\operatorname{dim} Z\left(Z_{G}(x)^{0}\right) \leq \operatorname{rank} G
$$

and equality holds if and only if $x$ is regular.

3. The following statements are equivalent:

(a) $x$ is regular.

(b) $Z_{G}(x)$ consists of polynomials in $x$.

(c) $Z_{G}(x)^{0}$ consists of polynomials in $x$.

(d) $Z_{G}(x)$ is abelian.

(e) $Z_{G}(x)^{0}$ is abelian.

(f) The minimal polynomial of $x$ is the characteristic polynomial of $x$.

(g) Different blocks have different eigenvalues in the Jordan form of $x$.

This more than takes care of groups of type $A_{n}$. For the rest of this section, we will be concerned with the orthogonal and symplectic groups, so we assume that char $K \neq 2$. At this point, we want to describe explicitly the map of Proposition 2.6. Let $G=S O_{n}(K)$ or $S p_{n}(K)$ and $\mathfrak{g}=\mathfrak{s}_{n}(K)$ or $\mathfrak{S}_{n}(K)$, respectively, Consider

$$
f(x)=(1-x)(1+x)^{-1}
$$

( 1 is the $n \times n$ identity matrix). This map takes unipotent elements of $G$ to nilpotent elements of $g([20])$. Its inverse is formally the same:

$$
f^{-1}(X)=(1-X)(1+X)^{-1}
$$

If $x$ is unipotent and $X$ nilpotent, then $f$ and $f^{-1}$ are polynomials in $x$ and $X$, respectively. Thus there is no problem in going from $G$ to $g$ and vice versa.

Proposition 3.2. Let $\mathfrak{g}=\mathfrak{g}_{n}(K)$ or $\mathfrak{g}_{n}(K)$ with char $K \neq 2$. Let $X \in \mathfrak{g}$ (not necessarily nilpotent) and let $k$ be the degree of the minimal polynomial of $x$. Then

$$
\operatorname{dim}(\{\text { polynomials in } x\} \cap \mathfrak{g})=\left[\frac{k}{2}\right]
$$

where $[n]$ is the greatest integer in $n$. 
Proof. If $s$ is the form, then $X \in \mathrm{g}$ means that

$$
X=-s^{-1} X^{t} s
$$

so

$$
X^{i}=(-1)^{i}\left(X^{i}\right)^{t} s
$$

and $X^{i} \in \mathrm{g}$ if and only if $i$ is odd.

Proposition 3.3. 1. Let $\mathfrak{g}=\mathfrak{S p}_{2 n}(K)$ or $\mathfrak{S}_{2 n+1}(K)$ with char $K \neq 2$. If $X \in \mathfrak{g}$ is a regular nilpotent element, then $X$ is regular in $\mathfrak{g l}_{2 n}(K)$ or $\mathfrak{S I}_{2 n+1}(K)$, respectively. Therefore,

$$
\jmath_{\mathfrak{g}}(X)=\{\text { polynomials in } X\} \cap \mathfrak{g} .
$$

2. Let $\mathfrak{g}=\mathfrak{S}_{\mathfrak{o}_{2 n}}(K)$ with char $K \neq 2$. If $X \in \mathfrak{g}$ is a regular nilpotent element, then $X$ is subregular in $\mathfrak{S l}_{2 n}(K)$. Hence, $\operatorname{dim}(\{$ polynomials in $X\} \cap \mathfrak{g})=n-1<$ rank $g$ and $\{$ polynomials in $X\} \cap \mathfrak{g}<z_{\mathfrak{g}}(X)$.

Proof. We use $e_{i j}$ to denote the matrix with 1 in the $(i, j)$ position and zeroes elsewhere. The argument for the first part consists of writing down the standard regular nilpotent element and seeing that it is regular in $\mathfrak{S l}_{2 n}(K)$ in the symplectic case and regular in $\mathfrak{S l}_{2 n+1}(K)$ in the orthogonal case. Then, Proposition 3.2 shows that \{polynomials in $X$ \} $\cap \mathrm{g}$ is the whole of $\mathfrak{z}_{\mathfrak{g}}(X)$. For part 2 , let $\mathfrak{g}=\mathfrak{s}_{2 n}(K)$ and let $X$ be the standard regular nilpotent element, i.e.

$$
X=\sum_{i=1}^{n-1}\left(e_{i, t+1}-e_{n+i+1, n+i}\right)+e_{n-1,2 n}-e_{n, 2 n-1} .
$$

Now the rank of $X$ is $2 n-2$ and if we look at the $n+1$ st basis vector, we see that $t^{2 n-1}$ is the minimal polynomial of $X$, thus $X$ is subregular in $\mathfrak{g l} \mathfrak{I}_{2 n}(K)$.

If $\mathrm{g}=\mathfrak{S o}_{2 n}(K)$ and $X$ is the standard regular nilpotent element, then an example of a $Y \in z_{\mathfrak{g}}(X)$ which is not a polynomial in $X$ is:

$$
Y=e_{1, n}-e_{2 n, n+1}+e_{n, n+1}-e_{1,2 n} \text {. }
$$

Then $X Y=Y X=0$. Thus is $Y$ where a polynomial in $X$, it would have to be a multiple of $X^{2 n-2}$, which cannot happen for two reasons: First, $Y^{2} \neq 0$; and second, $X^{2 n-2} \notin \mathrm{g}$.

If $X \in \mathfrak{S l}_{n}(K)$ is nilpotent, then the conjugacy class of $X$ (via $S L_{n}(K)$ ) is determined by the Jordan form of $X$, and thus by the sizes of the Jordan blocks. When we say that

$$
X \sim k_{1}, k_{2}, \ldots, k_{r}
$$


we mean that the Jordan form of $X$ has blocks of sizes $k_{1} \times k_{1}, k_{2} \times k_{2}$, etc. The usual conventions is that $k_{i} \geq k_{i+1}$ for all $i$. Then a nilpotent conjugacy class in $\mathfrak{S} \mathfrak{l}_{n}(K)$ is uniquely represented by a partition of $n$. The regular class is $n$, and the subregular class is $n-1,1$.

Let $X \in \mathfrak{S l}_{n}(K)$ be nilpotent with Jordan form $k_{1}, k_{2}, \cdots, k_{s}$, and let $r_{j}$ be the number of $k_{i}$ that are equal to $j$. Springer and Steinberg have shown that $X$ is conjugate (via $S L_{n}(K)$ ) to an element of $\mathfrak{g}_{n}(K)$ if and only if $r_{j}$ is even when $j$ is even, and $X$ is conjugate to an element of $\mathfrak{S p}_{n}(K)$ if and only if $r_{j}$ is even when $j$ is odd. In $\mathfrak{S}_{n}(K)$ and $\mathfrak{S}_{2 n+1}(K)$ the Jordan form determines the conjugacy class; in $\mathfrak{s}_{2 n}(K)$, two different conjugacy classes can have the same Jordan form, but then they are conjugate via $O_{2 n}(K)$ [16]. So to investigate $z_{g}(X)$ for $X$ nilpotent and $g$ orthogonal or symplectic it is sufficient to exhaust the possible Jordan forms for $X$.

LEMMA 3.4. Let $V$ be a finite dimensional vector space over $K$ (char $K \neq$ $2)$, and let $\mathfrak{g}=\mathfrak{s} \mathfrak{p}(V)$ or $\mathfrak{s} \mathfrak{D}(V)$. If $X \in \mathfrak{g}$ is nilpotent, then we can orthogonally decompose $V$ and $X$ :

$$
\begin{aligned}
& V=V_{1}+V_{2} \cdots+V_{r}, \\
& X=X_{1}+X_{2}+\cdots+X_{r},
\end{aligned}
$$

where $X_{i} \in \mathfrak{g}_{i}=\mathfrak{s} \mathfrak{p}\left(V_{i}\right)$ or $\mathfrak{s} \mathfrak{o}\left(V_{i}\right)$ respectively and either

(a) $X_{i}$ is regular in $\mathfrak{B} \mathfrak{l}\left(V_{i}\right)$ and $\mathrm{g}_{i}$, or

(b) $X_{i}$ has Jordan form $\lambda, \lambda$ where $\lambda$ is odd in the symplectic case and even in the orthogonal case.

Further, $\mathfrak{z}\left(\mathrm{z}_{\mathrm{g}_{i}}\left(X_{i}\right)\right)=\left\{\right.$ polynomials in $\left.X_{i}\right\} \cap \mathrm{g}_{i}$.

Proposition 3.5. Let $\mathfrak{g}=\mathfrak{S}_{\mathfrak{o}_{n}}(K)$ or $\mathfrak{g}_{n}(K)$ with char $K \neq 2$ and let $X \in \mathfrak{g}$ be nilpotent. Then

$$
z\left(z_{\mathrm{g}}(X)\right)=\{\text { polynomials in } X\} \cap \mathfrak{g}
$$

except in the case where $\mathfrak{g}=\mathfrak{S}_{2 m}(K)$ and $X$ has Jordan form $\lambda, \mu$ where $\lambda$ and $\mu$ are unequal and odd. In that case, \{polynomials in $X\} \cap \mathfrak{g}$ has codimension one in $z_{(}\left(z_{g}(X)\right)$. In any event,

$$
\operatorname{dim} z\left(z_{g}(X)\right) \leq \text { rank } g
$$

and equality holds if and only if $X$ is regular.

The proofs of 3.4 and 3.5 are straightforward. Note that 3.5 is sufficient to prove Theorem A for the classical groups. Let us say something about the exceptional case - which includes the case where $X \in \mathfrak{B}_{2 m}(K)$ is regular. For convenience, we change the form to sum of 
squares. Let

$$
X=\left[\begin{array}{c|c}
X_{1} & 0 \\
\hline 0 & X_{2}
\end{array}\right]
$$

where $X_{1} \in \mathfrak{S}_{\mathfrak{D}_{\lambda}}(K)$ and $X_{2} \in \mathfrak{S}_{\mathfrak{D}_{\mu}}(K)$ are regular nilpotent. Let

$$
W=\left[\begin{array}{c|c}
0 & A \\
\hline-A^{t} & 0
\end{array}\right]
$$

where $X_{1} A=0$ and $A X_{2}=0$. Then $W \in z\left(z_{\mathfrak{g}}(X)\right)$ but $W$ is not a polynomial in $X$.

THEOREM 3.7. Assume char $K \neq 2$

1. If $G=S p_{n}(K)$ and $x \in G$, then $Z\left(Z_{G}(x)^{0}\right)$ consists of polynomials in $x$.

2. If $G=S O_{n}(K)$ or $S p_{n}(K)$ and $x \in G$, then

$$
\operatorname{dim} Z\left(Z_{G}(x)^{0}\right) \leq \operatorname{rank} G
$$

and equality holds if and only if $x$ is regular. Therefore $x \in G$ is regular if and only if $Z_{G}(x)^{0}$ is abelian.

Proof. Let $x=s u$ be the Jordan decomposition of $x, H=Z_{G}(s)^{0}$, $\mathfrak{h}=z_{\mathrm{g}}(s), f$ the Cayley map, and $N=f(u)$.

1. $G=S p_{n}(K), \mathfrak{g}=\mathfrak{s} \mathfrak{p}_{n}(K)$.

Then $V=K^{n}=V_{1}+V_{2}+\cdots+V_{r}+V_{1}^{\prime}+V_{2}^{\prime}+\cdots+V_{r}^{\prime}$ where $\left.s\right|_{V_{i}}=\lambda_{i} I$ and $\left.s\right|_{V_{i}^{\prime}}=\lambda_{i}^{-1} I$.

(a) If no $\lambda_{i}$ is a $\lambda_{j}^{\prime}$, then

$$
\mathfrak{h}=\mathfrak{z}_{\mathfrak{g}}(s) \cong \mathfrak{g} \mathfrak{l}\left(V_{1}\right)+\cdots+\mathfrak{g} \mathfrak{l}\left(V_{r}\right)
$$

Now $Z_{G}(x)^{0}=Z_{H}(N)^{0}$ and the Lie algebra of this group is $z_{\mathfrak{h}}(N)$. So $z\left(z_{\mathfrak{h}}(N)\right)=z(h) \oplus(\{$ polynomials in $N\} \cap \mathfrak{h})=(\{$ polynomials in $s\} \cup$ \{polynomials in $N\}) \cap \mathfrak{h}$. Therefore $Z\left(Z_{G}(x)^{0}\right)$ consists of polynomials in $x$, because $s$ and $N$ are polynomials in $x$, and because for any affine group $A$,

$$
\mathcal{L}(Z(A)) \subseteq z(\mathcal{L}(A))
$$

(b) If some $\lambda_{i}=\lambda_{j}^{-1}$, then one gets a pair of symplectic algebras in $z_{\mathrm{g}}(s)-$ if $\lambda_{i}= \pm 1$, then one gets one symplectic algebra. Proposition 2.6 allows us to use the same argument as above. This completes the symplectic case. 
2. $G=S O_{n}(K)$. The situation here is similar to the symplectic case everything is easy unless $\lambda_{i}=\lambda_{j}^{-1}$ and then orthogonal algebras occur. For then if $N$ restricted to one of these algebras has just two unequal odd blocks, then - and only then - an element can appear in $Z\left(Z_{G}(x)^{0}\right)$ which is not a polynomial in $x$. Clearly the only time that $\operatorname{dim} Z\left(Z_{G}(x)^{0}\right)$ could equal rank $G$ is when $x$ is regular.

So now we have proved Theorem $\mathrm{C}$ and Theorems A and B for the classical groups. To finish the proofs of Theorems A and B, we need only work with the exceptional groups and algebras - this will be done in the next two sections.

4. Semiregular elements. Let $G$ be a reductive group and $g$ its Lie algebra. A unipotent element $x \in G$ (nilpotent element $X \in \mathfrak{g}$ ) is called semiregular if whenever a semisimple element $t \in G$ centralizes $x$ (or $X$ ), then $t \in Z(G)$. Regular elements are semiregular; however, we shall use semiregular mean semiregular and irregular. If char $K$ is good, then semiregular elements occur only in groups of type $D_{n}, E_{6}, E_{7}$, and $E_{8}$. In this section we will prove that if $\mathfrak{g}$ is $E_{6}, E_{7}$, or $E_{8}$, char $K$ is good for $\mathfrak{g}$, and $X \in \mathrm{g}$ is semiregular, then

$$
\operatorname{dim} z_{(}\left(z_{g}(X)\right)<\operatorname{rank} g .
$$

Thus, $z_{\mathfrak{g}}(X)$ is nonabelian. Unfortunately, our only proof of this is computational, so we omit the details which can be found in [8].

$E_{6}$ has only one semiregular class, while $E_{7}$ and $E_{8}$ each have two. These classes are usually denoted by $E_{6}\left(a_{1}\right), E_{7}\left(a_{1}\right), E_{7}\left(a_{2}\right), E_{8}\left(a_{1}\right)$, and $E_{8}\left(a_{2}\right)$. We use Elkington's paper [6] for representatives of these classes. For our numbering of the roots, the reader should take a glance at Table 1.

First, we handle $E_{6}\left(a_{1}\right)$. Here we take

$$
X=e_{\alpha_{1}}+e_{\alpha_{2}+\alpha_{3}}+e_{\alpha_{4}}+e_{\alpha_{5}}+e_{\alpha_{3}+\alpha_{6}}+e_{\alpha_{6}} .
$$

Proposition 4.1. Let $\mathrm{g}=E_{6}$ with $\operatorname{char} K \neq 2$ or 3 and let $X$ be as above. Then,

$$
\operatorname{dim} z\left(z_{g}(X)\right)=3<\operatorname{rank} g
$$

Next, we move to $E_{7}$. A representative for $E_{7}\left(a_{1}\right)$ is

$$
X=e_{\alpha_{1}}+e_{\alpha_{2}}+e_{\alpha_{3}+\alpha_{4}}+e_{\alpha_{5}}+e_{\alpha_{6}}+e_{\alpha_{4}+\alpha_{7}}+e_{\alpha_{7}} .
$$


This is the subregular class. A representative for $E_{7}\left(a_{2}\right)$ is

$$
Y=e_{\alpha_{1}}+e_{\alpha_{2}+\alpha_{3}+\alpha_{4}}+e_{\alpha_{3}}+e_{\alpha_{5}}+e_{\alpha_{6}}+e_{\alpha_{7}}+e_{\alpha_{4}+\alpha_{7}}
$$

$\operatorname{dim} z_{\mathfrak{g}}(Y)=11[4]$.

Proposition 4.2. Let $\mathrm{g}=E_{7}$ with char $K \neq 2$ or 3 and let $X$ and $Y$ be as above. Then,

$$
\operatorname{dim} \mathfrak{z}\left(z_{\mathfrak{g}}(X)\right)=5<\operatorname{rank} \mathfrak{g}
$$

and

$$
\operatorname{dim} z\left(z_{g}(Y)\right)=4<\text { rank } g .
$$

Finally, $\mathrm{g}=E_{8}$. A representative for $E_{8}\left(\alpha_{1}\right)$ is

$$
X=e_{\alpha_{1}}+e_{\alpha_{2}}+e_{\alpha_{3}}+e_{\alpha_{4}+\alpha_{5}}+e_{\alpha_{6}}+e_{\alpha_{7}}+e_{\alpha_{8}}+e_{\alpha_{5}+\alpha_{8}} ;
$$

$X$ is subregular. A representative for $E_{8}\left(\alpha_{2}\right)$ is

$$
Y=e_{\alpha_{1}}+e_{\alpha_{2}}+e_{\alpha_{3}+\alpha_{4}+\alpha_{5}}+e_{\alpha_{4}}+e_{\alpha_{5}+\alpha_{8}}+e_{\alpha_{6}}+e_{\alpha_{7}}+e_{\alpha_{8}} ;
$$

$\operatorname{dim}_{\jmath_{g}}(Y)=12[4]$.

Proposition 4.3. Let $\mathrm{g}=E_{8}$ with char $K \neq 2,3$, or 5 and let $X$ and $Y$ be as above. Then

$$
\operatorname{dim} z\left(z_{\mathfrak{g}}(X)\right)=5<\operatorname{rank} \mathfrak{g}
$$

and

$$
\operatorname{dim} z\left(z_{\mathfrak{g}}(Y)\right)=4<\operatorname{rank} g
$$

This completes the case of the semiregular elements. The next section deals with the rest of the irregular elements.

5. Non-semiregular elements. In this section we shall prove that if $g$ is an exceptional Lie algebra with char $K$ good for $g$ and if $X \in \mathfrak{g}$ is nilpotent and neither regular non semiregular, then $z_{\mathrm{g}}(X)$ is nonabelian. This will complete the proof of Theorem A. We will also prove Theorem B for $G_{2}$, which will complete the proof of Theorem B.

Let $\Psi$ be a root system. A subset $\Sigma \subseteq \Psi$ is called an integrally closed subsystem of $\Psi$ if $\Sigma$ itself is a root system and if whenever $n_{1} \alpha_{1}+\cdots+n_{r} \alpha_{r} \in \Psi$ where $n_{i} \in \mathbf{Z}$ and $\alpha_{l} \in \Sigma$, then $n_{1} \alpha_{1}+\cdots$ $+n_{r} \alpha_{r} \in \Sigma$. 
Let $G$ be a reductive group and $G_{1}$ a reductive subgroup of $G$ such that $G_{1}$ contains a maximal torus $T$ of $G$. Then we call $G_{1}$ an integrally closed subgroup of $G$ if the root system of $G_{1}$ relative to $T$ is an integrally closed subsystem of the root system of $G$ relative to $T$. We define integrally closed subalgebra in the analogous manner, although we must be careful in extending the roots to a larger Cartan subalgebra. Further, we need char $K$ to be good for the Lie algebra $g$.

If $\Sigma$ is an integrally closed subsystem of $\Psi$, then $\Sigma$ is called a maximal subsystem if there are no integrally closed subsystems properly between $\Sigma$ and $\Psi$. From this, we define maximal integrally closed reductive subgroup and subalgebra.

Proposition 5.1. Let $G$ be a reductive group and let $x \in G$ be unipotent. Then there is a reductive integrally closed subgroup $G_{1}$ of $G$ with the same rank as $G$ such that $x$ is regular or semiregular in $G_{1}$.

The proof is in [16] - note that it holds in all characteristics. The Lie algebra analogue is:

Proposition 5.2. Let $\mathrm{g}$ be a semisimple Lie algebra with char $K$ good for $\mathfrak{g}$ and let $X \in \mathfrak{g}$ be nilpotent. Then there is a semisimple integrally closed subalgebra $\mathrm{g}_{1}$ of $\mathrm{g}$ such that $X$ is regular or semiregular in $\mathrm{g}_{1}$. Further,

$$
\text { rank } \mathfrak{g}_{1} \leq \text { rank } \mathfrak{g} \text {. }
$$

The problem we will run into is the case where the ranks are the same. Anyway, the problem of determining nilpotent conjugacy classes in $g$ takes on a tractable form. First determine all the integrally closed subsystems of a given root system. Then determine which root systems can have semiregular elements. Finally, solve the conjugacy problem: Given $X_{i}$ regular or semiregular in $\mathfrak{g}_{l}(i=1,2)$ integrally closed subalgebras of $\mathfrak{g}$, then when are $X_{1}$ and $X_{2}$ conjugate in $\mathrm{g}$ ?

This was first done by Dynkin [4] in characteristic zero. The task of showing that the Dynkin results hold in all good characteristics has been completed by Pommerening $[10,11]$ (for a more complete list of citations, see $\S 1$ ).

Dynkin's method for determining the integrally closed subsystems of a root system $\Psi$ is as follows. Take the lowest root, $-\mu$, and attach it to the Dynkin diagram for $\Psi$ in the usual manner. That is, if $\alpha$ is a simple root, then $\alpha$ and $-\mu$ are joined by

$$
\frac{4(-\mu, \alpha)^{2}}{(-\mu,-\mu)(\alpha, \alpha)}
$$


lines. If $-\mu$ and $\alpha$ have different lengths, then one draws an arrow from the longer root to the shorter root. This gives the extended Dynkin diagram of $\Psi$. Table 1 shows the extended diagrams for all the irreducible root systems. From the extended diagram, one deletes one or more nodes. This gives the diagram of an integrally closed subsystem of $\Psi$. Repeating this process ultimately yields all the integrally closed subsystems of $\Psi$.

Proposition 5.3. (Dynkin). All the maximal integrally closed subsystems are obtained by omitting a root whose coefficient in the highest root is either one or a prime.

We proceed on the inductive assumption that we have proved Theorem $\mathfrak{a}$ for simpler systems - or if one prefers, induction is by dimension. Thus is $X$ is regular nilpotent in $g_{1}$ where $g_{1}$ is an integrally closed subsystem of $\mathfrak{g}$ which is not maximal, then we know that there is a semisimple subalgebra $\mathfrak{g}^{\prime}$ such that $\mathfrak{g}_{1} \subset \mathfrak{g}^{\prime} \subset \mathfrak{g}$ is a chain of integrally closed subalgebras. By induction, $z_{g^{\prime}}(X)$ is nonabelian. Of course if $X$ semiregular in $g$, then we already know (by $\S \S 3$ and 4) that

$$
\operatorname{dim} z\left(z_{g}(X)\right)<\operatorname{rank} g \text {. }
$$

So we are looking at the situation of a simple Lie algebra $g$ (with char $K$ good) and $X \in \mathfrak{g}_{1} \subset \mathfrak{g}$ where $X$ is regular nilpotent in $\mathfrak{g}_{1}$ an integrally closed subalgebra of $g$.

Proposition 5.4. Let $\mathrm{g}$ be a simple Lie algebra with char $K$ good for $\mathfrak{g}$. Suppose $X \in \mathfrak{g}$ is nilpotent such that $X$ is regular or semiregular in $\mathfrak{g}_{1}$, an integrally closed subalgebra of $\mathrm{g}$. Suppose further that rank $\mathrm{g}_{1}<$ rank $\mathrm{g}$. Then

$$
z\left(z_{g}(X)\right) \subseteq z_{g_{1}}(X)
$$

Therefore $\operatorname{dim} z_{(}\left(z_{\mathfrak{g}}(X)\right)<$ rank $\mathfrak{g}$.

Proof. We have to show that if $Y \in \mathfrak{g}-\mathfrak{g}_{1}$ centralizes $X$, then there is a $W \in z_{\mathrm{g}}(X)$ such that $[Y, W] \neq 0$. Let $\Psi$ be the root system for $\mathfrak{g}_{1}$. Since $z_{\mathfrak{g}_{1}}(X)$ is nilpotent, we can choose orderings for $\Psi$ and $\Psi_{1}$ such that

$$
\mathfrak{z}_{\mathfrak{g}_{1}}(X) \subseteq \mathfrak{g}_{1}^{+}=\operatorname{span}\left\{e_{\gamma} \mid \gamma \in \Psi_{1}^{+}\right\}
$$

and $\Psi_{1}^{+} \subset \Psi^{+}$. We use $\Delta$ to denote the base of $\Psi$ in this ordering, and we write

$$
Y=\sum_{\beta<0} a_{\beta} e_{\beta}+\sum_{\alpha \in \Delta} b_{\alpha} H_{\alpha}+\sum_{\beta>0} a_{\beta} e_{\beta}
$$

with $a_{\beta}, b_{\alpha} \in K$ and we label the three pieces of $Y$ as $Y^{-}, Y^{0}$, and $Y^{+}$. 
If there is a root $\delta \in \Psi-\Psi_{1}$ such that $a_{\delta} \neq 0$, then there is an $H \in \mathfrak{h}$ (the Cartan subalgebra for $g$ spanned by the $H_{\alpha}$ ) such that $\left[H, e_{\delta}\right]=e_{\delta}$ and $\left[H, \mathfrak{g}_{1}\right]=0$. Then $H$ will commute with $X$ but not with $Y$.

That leaves us with the case that both $Y^{-}$and $Y^{+} \in g_{1}$. As the Killing form is nondegenerate, we can write

$$
Y^{0}=H_{1}+H_{2}
$$

where $H_{l} \in \mathfrak{h}, H_{1} \in \mathfrak{g}_{1}$ and $\left[H_{2}, \mathfrak{g}_{1}\right]=0$. But then $Y^{-}+H_{1}+Y^{+} \in$ $\mathrm{z}_{\mathrm{g}_{1}}(X) \subseteq \mathrm{g}_{1}^{+}$, so $Y^{-}=H_{1}=0$. So what is really the case is that

$$
Y=Y^{+}+H_{2}
$$

where $Y^{+} \in \mathrm{g}_{1}^{+}$and $H_{2}$ is orthogonal to $\mathrm{g}_{1}$. Because $H_{2} \neq 0$ (otherwise $Y$ belongs to $\mathrm{g}_{1}$ ), there is a positive $\operatorname{root} \delta \in \Psi-\Psi_{1}$ such that $\delta$ is not orthogonal to $\mathrm{H}_{2}$. We take $\delta$ to have maximal height; that is

(i) $\left[H_{2}, e_{\delta}\right]=\lambda e_{\delta} \neq 0$ and

(ii) If ht $\nu>$ ht $\delta$, then $\left[H_{2}, e_{\nu}\right]=0$.

We now claim that $e_{\delta}$ is the $W$ that we want. First we must show that $e_{\delta}$ centralizes $X$ :

$$
\left[H_{2},\left[e_{\delta}, X\right]\right]=0
$$

by maximality of $\delta$ and the fact that $X$ is supported by positive roots. But

$$
\left[H_{2},\left[e_{\delta}, X\right]\right]=-\left[e_{\delta},\left[X, H_{2}\right]\right]-\left[X,\left[H_{2}, e_{\delta}\right]\right]=\left[X, \lambda e_{\delta}\right]
$$

because $H_{2}$ centralizes $X$. Therefore $\left[X, \lambda e_{\delta}\right]=0$ and so $e_{\delta} \in z_{\mathrm{g}}(X)$.

Next we show $\left[e_{\delta}, Y\right] \neq 0 .\left[e_{\delta}, Y\right]=\left[e_{\delta}, H_{2}+Y^{+}\right]=-\lambda e_{\delta}+$ higher root vectors. Hence $\left[e_{\delta}, Y\right] \neq 0$ and so $Y \notin z\left(z_{\mathrm{g}}(X)\right)$.

Finally, if $X$ is regular in $\mathfrak{g}_{1}$, then

$$
\operatorname{dim} z\left(z_{\mathfrak{g}_{1}}(X)\right)=\operatorname{rank} \mathfrak{g}_{1}
$$

and if $X$ is semiregular in $\mathfrak{g}_{1}$, then

$$
\operatorname{dim} z\left(z_{g_{1}}(X)\right)<\operatorname{rank} g_{1}
$$

In any event, then

$$
\operatorname{dim} \mathfrak{z}\left(z_{\mathfrak{g}}(X)\right)<\text { rank } \mathfrak{g} .
$$

The case where $g_{1}$ and $g$ have the same rank is much harder. In fact, it does not seem to be true that $z_{(}\left(z_{g}(X)\right) \subseteq g_{1}$ in that case. However we are still hopeful that it will turn out that $\operatorname{dim} z_{(}\left(z_{g}(X)\right) \leq$ rank $g$. What we did was to compute just enough of $z_{\mathrm{g}}(X)$ to show that it was nonabelian. Before we get to that, there is still one last thing that can be done. 
Proposition 5.5. Let $\mathrm{g}$ be a simple Lie algebra with char $K$ good for $\mathrm{g}$. Suppose $X \in \mathrm{g}$ is irregular and nilpotent such that $X$ is regular in a maximal integrally closed semisimple subalgebra $\mathrm{g}^{\prime}$ which is not simple. Then ${ }_{\mathfrak{g}}(X)$ is nonabelian.

Proof. Let $\Psi$ be a root system for $g$ with base $\Delta$. Then a base $\Delta^{\prime}$ for $\Psi^{\prime}$, the root system for $g^{\prime}$ is $\{-\mu\} \cup(\Delta-\{\beta\})$ for some simple root $\beta$ ( $\mu$ is the highest root). As $\Psi^{\prime}$ is reducible, there are at least two simple roots $\alpha_{1}$ and $\alpha_{2}$ that are connected to $\beta$ in the extended diagram (the case where one of these is $-\mu$ is permitted). So ignoring root lengths and possibly other roots connected to $\beta$, we have

$$
\begin{array}{lll}
\circ & \circ & -\circ \\
\alpha_{1} & \beta & \alpha_{2}
\end{array}
$$

It does no harm to assume that $X$ is standard regular in $\mathrm{g}^{\prime}$, that is,

$$
X=e_{-\mu}+\sum_{\alpha \in \Delta-\{\beta\}} e_{\alpha} .
$$

As $\Delta^{\prime}$ is reducible, $\Delta^{\prime}=\Delta_{1}^{\prime} \dot{\cup} \Delta_{2}^{\prime}$ with $\alpha_{i} \in \Delta_{i}^{\prime}$. Write $X=X_{1}+X_{2}$ where $X_{i}=\Sigma_{\alpha \in \Delta_{i}^{\prime}} e_{\alpha}$.

Now let $Y=e_{-\alpha_{1}-\beta}+a e_{-\alpha_{2}-\beta}$ where $a=-\left(N_{\alpha_{1},-\alpha_{1}-\beta} / N_{\alpha_{2},-\alpha_{2}-\beta}\right) \neq 0$.

Then $[X, Y]=0$ and clearly $\left[X, X_{i}\right]=0(i=1,2)$.

But $\left[X_{1}, Y\right]=N_{\alpha_{1},-\alpha_{1}-\beta} e_{-\beta} \neq 0$.

So $z_{g}(X)$ is nonabelian.

What remains is the following case: $g$ is a simple Lie algebra (char $K$ good), $\mathfrak{g}_{1} \subset \mathfrak{g}$ is a maximal integrally closed subalgebra of $\mathfrak{g}$ with $\mathfrak{g}_{1}$ simple; $X$ is regular nilpotent in $g_{1}$ such that $X$ is not conjugate to an element in a "smaller" subalgebra (i.e. one that is integrally closed but not maximal). These are the possibilities: (cf. Table 1)

(a) $\mathfrak{g}=G_{2}$

(b) $\mathrm{g}=F_{4}$

$X=e_{-\mu}+e_{\beta}\left(\right.$ type $\left.A_{2}\right)$

$X=e_{-\mu}+e_{\alpha}+e_{\beta}+e_{\gamma}\left(\right.$ type $\left.B_{4}\right)$

(c) $\mathrm{g}=E_{6}$

Any irreducible integrally closed subsystem which is maximal is $E_{6}$, so nothing happens here.

(d) $\mathrm{g}=E_{7}$

$X=e_{\alpha_{1}}+e_{\alpha_{2}}+e_{\alpha_{3}}+e_{\alpha_{4}}+e_{\alpha_{5}}+e_{\alpha_{6}}+e_{-\mu}\left(\right.$ type $\left.A_{7}\right)$

We will show that $X$ is conjugate to an element semiregular in a system of type $E_{6}$, so nothing happens here either. 
(e) $\mathfrak{g}=E_{8}$

(i) $X=e_{-\mu}+e_{\alpha_{1}}+e_{\alpha_{2}}+e_{\alpha_{3}}+e_{\alpha_{4}}+e_{\alpha_{5}}+e_{\alpha_{6}}+e_{\alpha_{7}}$ (type $A_{8}$ )

(ii) $X=e_{-\mu}+e_{\alpha_{1}}+e_{\alpha_{2}}+e_{\alpha_{3}}+e_{\alpha_{4}}+e_{\alpha_{5}}+e_{\alpha_{6}}+e_{\alpha_{8}}$ (type $D_{8}$ ).

First we tackle $\mathrm{g}=G_{2} . X=e_{-\mu}+e_{\beta}$. Dynkin has shown that $X$ is subregular, so $\operatorname{dim} z_{\mathrm{g}}(X)=4$. Now $X$ is conjugate to $Y=e_{-\mu}+e_{\alpha}$ to which we could apply Proposition 5.5, but that would not help us with Theorem B. The following result can be checked by computation.

Proposition 5.6. Let $\mathrm{g}=G_{2}$ with char $K \neq 2$ or 3 , and let $X=e_{-\mu}+$ $e_{\beta}$. Then a basis for $z_{\mathfrak{g}}(X)$ is $\left\{X, e_{-\alpha}, e_{-2 \alpha-\beta}, e_{-3 \alpha-\beta}\right\}$ and a basis for $z\left(z_{\mathfrak{g}}(X)\right)$ is $\left\{X, e_{-3 \alpha-\beta}\right\}$.

In $G_{2}$, there are no irregular semiregular elements. The only other integrally closed subsystem of rank 2 is $A_{1}+A_{1}$ whose regular element is the $Y$ above. By Dynkin's tables $X$ and $Y$ are conjugate, so $\operatorname{dim} z\left(z_{g}(Y)\right)$ $=2$. Note that for $X$ and $Y$, the double centralizer has dimension 2 which equals the rank.

Proposition 5.7. Let $G=G_{2}$ with char $K \neq 2$ or 3. If $x \in G$, then

$$
\operatorname{dim} Z\left(Z_{G}(x)^{0}\right) \leq \operatorname{rank} G=2
$$

Equality can occur only when

1. $x$ is regular, or

2. $x$ is subregular, but not semisimple.

Proof. If $x$ is subregular semisimple, then $Z_{G}(x)^{0}$ is reductive of rank 2 and dimension 4 , hence $\operatorname{dim} Z\left(Z_{G}(x)^{0}\right)=1$. If $x=s u$ is the Jordan decomposition of a subregular element $x$ which is neither unipotent nor semisimple, then $s$ and $u$ belong to $Z\left(Z_{G}(x)^{0}\right)$ and so $\operatorname{dim} Z\left(Z_{G}(x)^{0}\right)=2$. Proposition 5.6 handles subregular unipotent elements and Proposition 5.4 handles everything else.

This finishes the proof of Theorem B and proves Theorem A for $G_{2}$. We now move to $F_{4}=\mathrm{g}$. The element $X$ regular in a system of type $B_{4}$ is subregular in $F_{4}$. Thus $\operatorname{dim}_{z_{\mathfrak{g}}}(X)=6$.

Proposition 5.8. Let $\mathrm{g}=F_{4}$ with char $K \neq 2$ or 3 and let

$$
X=e_{-\mu}+e_{\alpha}+e_{\beta}+e_{\gamma} .
$$

Then the following six elements form a basis for ${ }_{\mathfrak{g}}(X)$ :

$$
\begin{gathered}
X, e_{-\delta}, e_{-\beta-2 \gamma-2 \delta}, e_{\alpha+\beta+\gamma}+a e_{\beta+2 \gamma}+b e_{-\mu}, \\
e_{\alpha+2 \beta+2 \gamma}+c e_{-\alpha-2 \beta-2 \gamma-2 \delta}, \text { and } e_{-\alpha-\beta-\gamma-\delta}+d e_{-\beta-2 \gamma-\delta},
\end{gathered}
$$


for appropriate $a, b, c, d \in K$. Moreover,

$$
\operatorname{dim} z\left(z_{\mathfrak{g}}(X)\right) \leq 3<\operatorname{rank} \mathfrak{g} .
$$

The proof is computational. To find the exact values of $a, b, c$, and $d$ (and then the exact dimension of $z\left(z_{g}(X)\right)$ ), one must compute a number of structure constants. In any event, $a= \pm 2$ and $b, c$, and $d$ are \pm 1 . More detail can be found in [8]. This proves Theorem $\mathrm{A}$ for $F_{4}$.

As we have said, $E_{6}$ has no irreducible maximal integrally closed subsystems, so the proof of Theorem $\mathrm{A}$ is complete for $E_{6}$ as well. To handle $E_{7}$, we have to describe the Dynkin diagram of a nilpotent element in a simple Lie algebra $g$.

If $\mathrm{g}$ is simple and $X \in \mathfrak{g}$ is nilpotent, then there is a $\{X, Y, H\} \subseteq \mathfrak{g}$ with $X$ and $Y$ nilpotent and $H$ semisimple so that $[X, Y]=H,[H, X]=$ $2 X$ and $[H, Y]=-2 Y$. By taking the Dynkin diagram for $g$ and putting the number $\alpha_{i}(H)$ at the $\alpha_{i}$ node, we get the Dynkin diagram for $X$. By applying the Weyl group $W$ to the base, we can always arrange matters so that the numbers $\alpha_{i}(H)$ are 0,1 , or 2 . A nilpotent element is regular if and only if it has a 2 at every node in its diagram.

In our case, $\mathrm{g}=E_{7}$ (char $K \neq 2$ or 3 ), and $X$ is regular in a subsystem of type $A_{7}$, with base $\alpha_{1}, \alpha_{2}, \alpha_{3}, \alpha_{4}, \alpha_{5}, \alpha_{6},-\mu$. Thus $\alpha_{1}(H)=\alpha_{2}(H)=$ $\cdots=-\mu(H)=2$. Since $\mu$ has an $\alpha_{7}$ term, we can see that $\alpha_{7}(H)=-16$. If we write the diagram of $X$ with just the numbers $\alpha_{i}(H)$, we have

$$
\begin{gathered}
222222 \\
-16
\end{gathered}
$$

If $w_{i}$ is reflection in $\alpha_{i}$, then by applying $w_{7}$, we get

$$
\begin{gathered}
222-1422 \\
16
\end{gathered}
$$

Continuing, we eventually get

$$
\begin{gathered}
020202 \\
0
\end{gathered}
$$

Consulting the Dynkin tables (in [4] or [6]), we see that this is the diagram for $E_{6}\left(a_{1}\right)$. That is, $X$ is conjugate to an element which is semiregular in a subsystem of type $E_{6}$. So by Propositions 4.1 and 5.4, we see

$$
\operatorname{dim} z\left(z_{g}(X)\right) \leq 3 .
$$

Thus we have proven:

Proposition 5.9. Let $\mathrm{g}=E_{6}$ or $E_{7}$ with char $K \neq 2$ or 3. If $X \in \mathrm{g}$ is nilpotent and irregular, then $\mathrm{z}_{\mathrm{g}}(X)$ is nonabelian. 
That finishes the proof of Theorem $\mathrm{A}$ for $E_{6}$ and $E_{7}$, leaving us only with $\mathfrak{g}=E_{8}$. Unfortunately, the regular elements in the $A_{8}$ and $D_{8}$ subsystems are not conjugate to anything in a smaller subsystem. Hence, what we do is compute a large enough part of the centralizers to show that they are nonabelian. This is presented in [8] with all the details.

To simplify notation, we will use the 8-tuple $\left(n_{1} n_{2} n_{3} n_{4} n_{5} n_{6} n_{7} n_{8}\right)$ to denote the root $\beta=\sum_{i=1}^{8} n_{i} \alpha_{i}$ when $n_{i} \geq 0$. For $-\beta$, we write $-\left(n_{1} n_{2} n_{3} n_{4} n_{5} n_{6} n_{7} n_{8}\right)$. Since $n_{i}$ is at most 6 , this is unambiguous. The proofs of the next two results are simply computations.

First we handle the $A_{8}$ subsystem. So

$$
X=e_{-\mu}+e_{\alpha_{1}}+e_{\alpha_{2}}+e_{\alpha_{3}}+e_{\alpha_{4}}+e_{\alpha_{5}}+e_{\alpha_{6}}+e_{\alpha_{7}} .
$$

Dynkin's tables tell us that $\operatorname{dim} \jmath_{g}(X)=24$.

Proposition 5.10. Let $\mathrm{g}=E_{8}$ with char $K \neq 2,3$, or 5 and let $X$ be as above. Let

$$
Y=e_{-(12345423)}+e_{(11111100)}-e_{(01111110)}
$$

and

$$
\begin{aligned}
Z= & e_{(12345422)}-e_{-(1111101)}+2 e_{-(01112101)}-e_{-(01111111)} \\
& -3 e_{-(00122101)}+e_{-(00112111)}-e_{-(00012211)} .
\end{aligned}
$$

Then $Y$ and $Z$ both centralize $X$ but not each other and so $z_{g}(X)$ is nonabelian.

Lastly, we have the $D_{8}$ case. So

$$
X=e_{-\mu}+e_{\alpha_{1}}+e_{\alpha_{2}}+e_{\alpha_{3}}+e_{\alpha_{4}}+e_{\alpha_{5}}+e_{\alpha_{6}}+e_{\alpha_{8}}
$$

From Dynkin's tables, we see that $\operatorname{dim} z_{g}(X)=16([4]$ or [6]).

Proposition 5.11. Let $\mathrm{g}=E_{8}$ with char $K \neq 2,3$, or 5 and let $X$ be as above. Let

$$
Y=e_{-(12345422)}-e_{(1111101)}+e_{(01112101)}-e_{(00122101)}
$$

and

$$
Z=e_{-(12344322)}-e_{(11122101)}+e_{(01222101)} .
$$

Then $Y$ and $Z$ both centralize $X$, but not each other. Therefore, $z_{\mathfrak{g}}(X)$ is nonabelian.

This finishes the proof of Theorem A. 
152

JOHN F. KURTZKE, JR.

TABLE 1

Extended Dynkin diagrams for the irreducible root systems

$A_{n}(n \geq 1)$

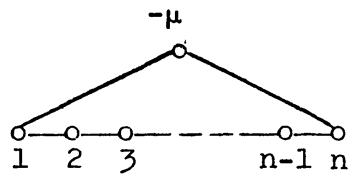

$B_{n}(n \geq 2)$

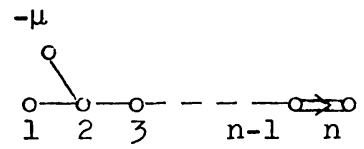

$$
\mu=\alpha_{1}+\alpha_{2}+\ldots+\alpha_{n}
$$

$\mu=\alpha_{1}+2 \alpha_{2}+2 \alpha_{3}+\ldots+2 \alpha_{n-1}+2 \alpha_{n}$

$c_{n}(n \geq 3)$

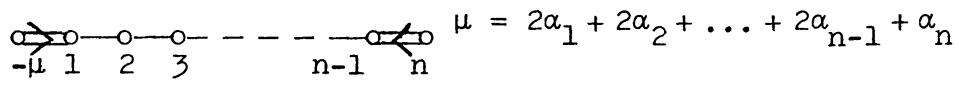

$D_{n}(n \geq 4)$

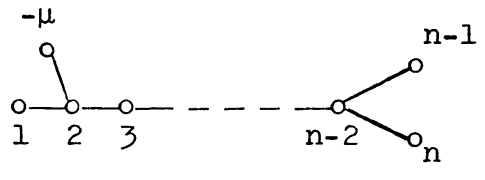

$\mu=\alpha_{1}+2 \alpha_{2}+2 \alpha_{3}+\ldots+2 \alpha_{n-2}$

$+\alpha_{n-1}+\alpha_{n}$

$G_{2}$

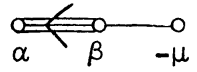

$\mu=3 \alpha+2 \beta$

$\mathrm{F}_{4}$

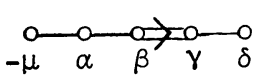

$\mu=2 \alpha+3 \beta+4 \gamma+2 \delta$

$\mathrm{E}_{6}$

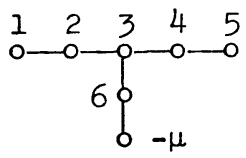

$\mu=\alpha_{1}+2 \alpha_{2}+3 \alpha_{3}+2 \alpha_{4}+\alpha_{5}+2 \alpha_{6}$

$E_{7}$

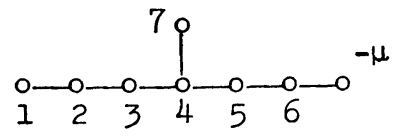

$\mu=\alpha_{1}+2 \alpha_{2}+3 \alpha_{3}+4 \alpha_{4}+3 \alpha_{5}$

$+2 \alpha_{6}+2 \alpha_{7}$

$E_{8}$

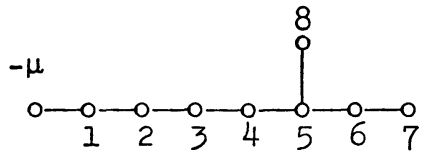

$\mu=2 \alpha_{1}+3 \alpha_{2}+4 \alpha_{3}+5 \alpha_{4}$

$+6 \alpha_{5}+4 \alpha_{6}+2 \alpha_{7}+3 \alpha_{8}$ 
ACKNOWLEDGEMENTS. This paper is a revision of the author's Ph.D. dissertation at U.C.L.A. under the direction of Professor Robert Steinberg. The author is very much indebted to Professor Steinberg and wishes to thank him here. The author also wishes to thank Professor Mario Borelli for reading an earlier version of this paper, and the referee for his suggestions.

\section{REFERENCES}

1. P. Bala and R. W. Carter, Classes of unipotent elements in simple algebraic groups, I, Math. Proc. Camb. Phil. Soc., 79 (1976), 401-425.

2. Classes of unipotent elements in simple algebraic groups, II, Math. Proc. Camb. Phil. Soc., 80 (1976), 1-18.

3. C. Chevalley, Seminaire Chevalley, (Paris: Ecole Norm. Sup. 1956-1958).

4. E. B. Dynkin, Semisimple subalgebras of semisimple Lie algebras, Amer. Math. Soc. Transl., (2) 6 (1957), 111-245.

5. A. G. Elasvili, Centralizers of nilpotent elements in semisimple Lie algebras, Trudy Tbilisskogo Mat. Inst., 46 (1975), 109-132 (Russian).

6. G. B. Elkington, Centralizers of unipotent elements in semisimple algebraic groups, J. Algebra, 23 (1972), 137-163.

7. J. E. Humphreys, Introduction to Lie Algebras and Representation Theory, (New York-Heidelberg-Berlin: Springer-Verlag, 1972).

7a. L_ Linear Algebraic Groups, (New York-Heidelberg-Berlin: Springer-Verlag, 1975).

8. J. F. Kurtzke, Jr., The Centralizers of an Irregular Element in a Reductive Algebraic Group, Ph.D. thesis, U.C.L.A., 1978.

9. B. Lou, Centralizers of Regular Unipotent Elements in Chevalley Groups, Ph.D. thesis, U.C.L.A., 1967.

10. K. Pommerening, Über die unipotenten Klasses reduktiver Gruppen, J. Algebra, 49 (1977), 525-536.

11. __ Über die unipotenten Klassen reduktiver Gruppen II, J. Algebra, 65 (1980), 373-398.

12. R. W. Richardson, Jr., Conjugacy classes in Lie algebras and algebraic groups, Annals of Math., 86 (1967), 1-15.

13. T. A. Springer, A note on centralizers in semi-simple groups, Nederl. Akad. Wetensch. Proc. Ser. A, 69 (1966), 75-77.

14. __ Some arithmetrical results on semisimple Lie algebras, Publ. Math. IHES no. 30 (1966), 115-141.

15. _ The unipotent variety of a semisimple group, in Algebraic Geometry, Papers Presented at the Bombay Colloquium 1968: Proceedings of Symposia in Pure Mathematics vol. 29 (Providence, R.I.: American Mathematical Society, 1975), 373-391.

16. T. A. Springer, R. Steinberg, Conjugacy classes, in Seminar on Algebraic Groups and Related Finite Groups, Lecture Notes in Mathematics vol. 131 (New York-HeidelbergBerlin: Springer-Verlag, 1970).

17. R. Steinberg, Regular elements of semisimple algebraic groups, Publ. Math. IHES no. 25 (1965), 49-80. 
18. Conjugacy Classes in Algebraic Groups, Lecture Notes in Mathematics vol. 366 (New York-Heidelberg-Berlin: Springer-Verlag, 1974).

19. U. Stuhler, Unipotente und nilpotente Klassen in einfachen Gruppen und Liealgebren von Typ $G_{2}$, Nederl. Akad. Wetensch. Proc. Ser. A, 74 (1971), 365-378.

20. H. Weyl, Classical Groups (Princeton: Princeton University Press, 1946).

Received January 25, 1980 and in revised form November 30, 1981.

UNIVERSITY OF NOTRE DAME

NOTRE DAME, IN 46556 


\section{PACIFIC JOURNAL OF MATHEMATICS \\ EDITORS}

DONALD BABBITT (Managing Editor)

University of California

Los Angeles, CA 90024

\section{Hugo Rossi}

University of Utah

Salt Lake City, UT 84112

C. C. Moore and Arthur Ogus

University of California

Berkeley, CA 94720
J. DugundiI

Department of Mathematics

University of Southern California

Los Angeles, CA 90089-1113

R. FINN and H. SAMELSON

Stanford University

Stanford, CA 94305

ASSOCIATE EDITORS
R. ARens
E. F. BECKENBACH
B. H. NeumanN
F. WOLF
K. YoshidA (1906-1982)

\section{SUPPORTING INSTITUTIONS}

UNIVERSITY OF ARIZONA

UNIVERSITY OF BRITISH COLUMBIA

CALIFORNIA INSTITUTE OF TECHNOLOGY

UNIVERSITY OF CALIFORNIA

MONTANA STATE UNIVERSITY

UNIVERSITY OF NEVADA, RENO

NEW MEXICO STATE UNIVERSITY

OREGON STATE UNIVERSITY
UNIVERSITY OF OREGON

UNIVERSITY OF SOUTHERN CALIFORNIA

STANFORD UNIVERSITY

UNIVERSITY OF HAWAII

UNIVERSITY OF TOKYO

UNIVERSITY OF UTAH

WASHINGTON STATE UNIVERSITY

UNIVERSITY OF WASHINGTON 


\section{Pacific Journal of Mathematics}

Vol. 104, No. 1

May, 1983

Nestor Edgardo Aguilera and Eleonor Ofelia Harboure de Aguilera, On

the search for weighted norm inequalities for the Fourier transform $\ldots \ldots .1$

Jin Akiyama, Frank Harary and Phillip Arthur Ostrand, A graph and its complement with specified properties. VI. Chromatic and achromatic numbers ......................................... 15

Bing Ren Li, The perturbation theory for linear operators of discrete type . . . 29

Peter Botta, Stephen J. Pierce and William E. Watkins, Linear

transformations that preserve the nilpotent matrices .............. 39

Frederick Ronald Cohen, Ralph Cohen, Nicholas J. Kuhn and Joseph

Alvin Neisendorfer, Bundles over configuration spaces .......... 47

Luther Bush Fuller, Trees and proto-metrizable spaces . . . . . . . . . . 55

Giovanni P. Galdi and Salvatore Rionero, On the best conditions on the

gradient of pressure for uniqueness of viscous flows in the whole space . . 77

John R. Graef, Limit circle type results for sublinear equations $\ldots \ldots \ldots \ldots 85$

Andrzej Granas, Ronald Bernard Guenther and John Walter Lee,

Topological transversality. II. Applications to the Neumann problem for

$y^{\prime \prime}=f\left(t, y, y^{\prime}\right) \ldots \ldots \ldots \ldots \ldots \ldots \ldots \ldots \ldots \ldots \ldots \ldots \ldots \ldots \ldots . \ldots 5$

Richard Howard Hudson and Kenneth S. Williams, Extensions of

theorems of Cunningham-Aigner and Hasse-Evans . . . . . . . . . . . 111

John Francis Kurtzke, Jr., Centralizers of irregular elements in reductive algebraic groups

James F. Lawrence, Lopsided sets and orthant-intersection by convex

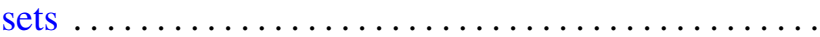

Åsvald Lima, G. H. Olsen and U. Uttersrud, Intersections of $M$-ideals and

$G$-spaces

Wallace Smith Martindale, III and C. Robert Miers, On the iterates of derivations of prime rings

Thomas H. Pate, Jr, A characterization of a Neuberger type iteration procedure that leads to solutions of classical boundary value problems

Carl L. Prather and Ken Shaw, Zeros of successive iterates of multiplier-sequence operators

Billy E. Rhoades, The fine spectra for weighted mean operators

Rudolf J. Taschner, A general version of van der Corput's difference theorem

Johannes A. Van Casteren, Operators similar to unitary or selfadjoint ones 